University of New Hampshire

University of New Hampshire Scholars' Repository

Physics Scholarship

Physics

2-1994

\title{
COMPTEL measurements of the gamma-ray burst GRB 930131
}

James M. Ryan

University of New Hampshire, James.Ryan@unh.edu

K Bennett

ESTEC

W Collmar

Max-Planck-Institut für extraterrestriche Physik

A Connors

University of New Hampshire - Main Campus

Gerald J. Fishman

NASA Marshall Space Flight Center

See next page for additional authors

Follow this and additional works at: https://scholars.unh.edu/physics_facpub

Part of the Astrophysics and Astronomy Commons

\section{Recommended Citation}

J. Ryan, K. Bennett, W. Collmar, A. Connors, G. J. Fishman, J. Greiner, L. O. Hanlon, W. Hermsen, R. M. Kippen, C. Kouveliotou, L. Kuiper, G. Lichti, J. Macri, J. Mattox, M. McConnell, B. McNamara, C. Meegan, V. Schoenfelder, R. van Dijk, M. Varendorff, W. R. Webber, and C. Winkler, 'COMPTEL measurements of the gamma-ray burst GRB 930131', The Astrophysical Journal, vol. 422, p. L67, Feb. 1994.

This Article is brought to you for free and open access by the Physics at University of New Hampshire Scholars' Repository. It has been accepted for inclusion in Physics Scholarship by an authorized administrator of University of New Hampshire Scholars' Repository. For more information, please contact Scholarly.Communication@unh.edu. 


\section{Authors}

James M. Ryan, K Bennett, W Collmar, A Connors, Gerald J. Fishman, J Greiner, L O. Hanlon, W Hermsen, R M. Kippen, C Kouveliotou, L Kuiper, G G. Lichti, John R. Macri, J. Mattox, Mark L. McConnell, B McNamara, C Meegan, V. Schonfelder, R VanDijk, M Varendorff, W Webber, and C Winkler 
ThE ASTROPHYSICAL JoURNAL, 422:L67-L70, 1994 February 20

(1) 1994. The American Astronomical Society. All rights reserved. Printed in U.S.A.

\title{
COMPTEL MEASUREMENTS OF THE GAMMA-RAY BURST GRB 930131
}

\author{
J. Ryan, ${ }^{1}$ K. Bennett, ${ }^{2}$ W. Collmar ${ }^{3}$ A. Connors, ${ }^{1}$ G. J. Fishman, ${ }^{4}$ J. Greiner, ${ }^{3}$ \\ L. O. Hanlon, ${ }^{2}$ W. Hermsen, ${ }^{5}$ R. M. KipPen, ${ }^{1}$ C. Kouveliotou, ${ }^{4}$ L. KuiPer, ${ }^{5}$ \\ G. Lichti, ${ }^{3}$ J. Macri, ${ }^{1}$ J. Mattox, ${ }^{6}$ M. McConnell, ${ }^{1}$ B. McNamara, ${ }^{7}$ \\ C. MeEgan, ${ }^{4}$ V. Schönfelder, ${ }^{3}$ R. VAN DiJK, ${ }^{5}$ M. VARendorfF, ${ }^{1}$ \\ W. R. WEBBER, ${ }^{1}$ AND C. WINKLER ${ }^{2}$ \\ Received 1993 October 4 ; accepted 1993 December 2
}

\begin{abstract}
On 1993 January 31 at 1857:12 UT, the Imaging Compton Telescope COMPTEL onboard the Compton Gamma Ray Observatory (CGRO) detected the cosmic $\gamma$-ray burst GRB 930131. COMPTEL's MeV imaging capability was employed to locate the source to better than $2^{\circ}$ ( $1 \sigma$ error radius) within $7 \mathrm{hr}$ of the event, initiating a world-wide search for an optical and radio counterpart. The maximum likelihood position of the burst from the COMPTEL data is $\alpha_{2000}=12^{\mathrm{h}} 18^{\mathrm{m}}, \delta_{2000}=-9^{\circ} 42^{\prime}$, consistent with independent CGROBATSE and EGRET locations as well as with the triangulation annulus constructed using BATSE and Ulysses timing data. The combined COMPTEL and EGRET burst data yield a better estimate of the burst location: $\alpha_{2000}=12^{\mathrm{h}} 12^{\mathrm{m}}$ and $\delta_{2000}=-10^{\circ} 21^{\prime}$, with a $1 \sigma$ error radius of $32^{\prime}$. In COMPTEL's energy range, this burst was short, consisting of two separate spikes occurring within a $\sim 1$ s interval with a low intensity tail for $\sim 1 \mathrm{~s}$ after the second spike. No statistically significant flux is present for a $30 \mathrm{~s}$ period after the main part of the burst. This is consistent with the EGRET data. The COMPTEL telescope events indicate a hard, power-law emission extending to beyond $10 \mathrm{MeV}$ with a spectral index of $-1.8 \pm 0.4$. The rapid fluctuations and high intensities of the $\gamma$-ray flux $>10 \mathrm{MeV}$ place the burst object no farther than 250 pc if the burst emission is not beamed.
\end{abstract}

Subject heading: gamma rays: bursts

\section{INTRODUCTION}

Given our present level of understanding, a detection of a $\gamma$-ray burst at any other wavelength would greatly enhance our understanding of the burst phenomena (Schaefer et al. 1994). Searchers over a variety of timescales with sensitive instruments are necessary to draw meaningful conclusions about the luminosity of any optical counterpart. Quick, deep searches require precise, accurate, and timely positions for $\gamma$-ray bursts. Interplanetary network triangulations are capable of arcminute locations but are generally available only days after the event. Quick searches at other wavelengths, therefore, rely upon wide-FOV instruments operating in real time with the eventual occurrence of a burst within the FOV of the optical instrument. Projects or campaigns of this type have been summarized by e.g., Vanderspek, Doty, \& Ricker (1992) and Greiner et al. (1993).

The BATSE instrument (Fishman et al. 1989) is capable of obtaining locations on short notice with $>4^{\circ}$ (radius) error boxes. However, if a burst can be localized with confidence to be within the FOV of a Schmidt camera, then instruments larger than $60 \mathrm{~cm}$ can reach sensitivities better than 19th mag in $1 \mathrm{hr}$ exposures. The required precision and accuracy of burst

\footnotetext{
${ }^{1}$ Space Science Center, University of New Hampshire, Durham, NH 03824.

${ }^{2}$ Astrophysics Division, ESTEC, Noordwijk, The Netherlands.

${ }^{3}$ Max-Planck Institut für Extraterrische Physik, Garching, Germany

${ }^{4}$ Space Sciences Laboratory, Marshall Space Flight Center, Huntsville, AL 38512 .

${ }^{5}$ SRON, Leiden, 2300 RA Leiden, The Netherlands.

${ }^{6}$ NASA/Goddard Space Flight Center, Code 662, Greenbelt, MD 20771; and Computer Sciences Corporation, Greenbelt, MD 20771.

7 Astronomy Department, New Mexico State University, Las Cruces, NM 88003.
}

locations that enable the use of these telescopes can be obtained with COMPTEL (Schönfelder et al. 1993).

The burst localization process involves only the doublescatter (or telescope) mode of COMPTEL. In the telescope mode, COMPTEL "focuses" $\gamma$-rays with a nominal source location accuracy, which, depending on source strength, can be as good as 0.5 (e.g., GRB 910503 and GRB 910814; Hanlon et al. 1993) with an energy resolution better than 10\% FWHM (at $662 \mathrm{keV})$.

The observation of GRB 930131 is significant because of the peak flux of the burst, its hard spectrum (extending beyond 10 $\mathrm{MeV}$ ), its temporal evolution, and the fact that it was located within $7 \mathrm{hr}$ of the event (Ryan, Kippen, \& Varendorff 1993). This counterpart search demonstrates the utility of COMPTEL in multiwavelength observations of $\gamma$-ray bursts.

\section{DATA AND RESULTS}

Figure 1 shows the count rate of the telescope events recorded by COMPTEL and the rates of two individual D2 detectors. As seen in the data, the burst consists of two spikes within a $1 \mathrm{~s}$ interval. However, both BATSE, EGRET, and COMPTEL ( $>600 \mathrm{keV}$ in the "burst mode" spectrometer data) detect $\gamma$-rays beyond this $1 \mathrm{~s}$. The major difficulty in measuring the light curve of an intense burst with COMPTEL is the limited capacity of the telemetry and event processing systems (Schönfelder et al. 1993). High rates of individual detector modules $\left(>10^{5} \mathrm{~s}^{-1}\right)$ also may affect the instrument livetime.

Because of the complexity of the burst and performance of the instrument during the event, we have subdivided the burst into several intervals as the basis for the following discussion. The moment of the internal BATSE trigger (1857:11.682 UT) 
GRB930131 - COMPTEL Telescope Time History

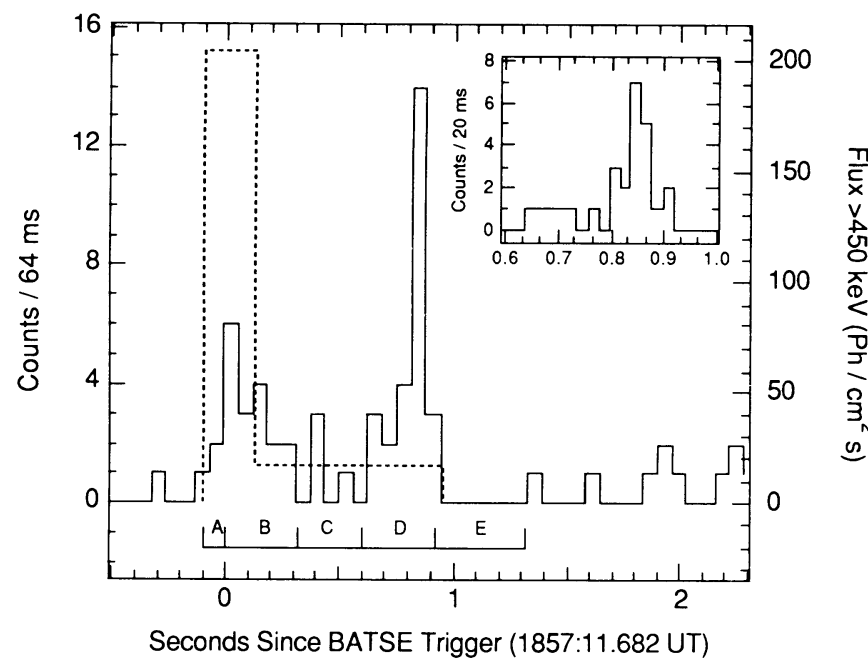

FIG. 1.-The intensity-time profile of GRB 930131 from COMPTEL telescope data. The indicated time intervals are discussed in the text. The left vertical axis refers to the telescope count rate data (solid line), while the right vertical axis refers to the two bin histogram obtained from two omnidirectional D2 detectors $>450 \mathrm{keV}$ (dashed line). The inset is a $20 \mathrm{~ms}$ time resolution light curve in time interval $\mathrm{D}$

serves as a reference time. The intervals are defined as follows:

A. $-1857: 11.582-1857: 11.682$ UT, the onset of the burst as measured by COMPTEL, the time of the most intense $\gamma$-ray flux. Livetime fraction $\sim 0 \%$.

B.- $1857: 11.682-1857: 12.0 \mathrm{UT}$, the $300 \mathrm{~ms}$ trailing edge of the main spike.

C. - 1857:12.0-1857:12.28 UT, a $300 \mathrm{~ms}$ interval between spikes where there is little activity. Livetime fraction $\sim 98 \%$.

D. - 1857:12.28-1857:12.602 UT, contains a second spike lasting $50 \mathrm{~ms}$.

E.-1857:12.602-1857:13.0 UT, a $300 \mathrm{~ms}$ interval during which COMPTEL can make no measurements. Livetime fraction $=0 \%$.

Average livetime information is recorded on a $16.4 \mathrm{~s}$ basis. The intense $1 \mathrm{~s}$ of the burst was fully contained in such a $16.4 \mathrm{~s}$ interval. An analysis of the various components of the livetime indicates that the effective livetime for the intense $1 \mathrm{~s}$ of the burst was $12 \%$. The instantaneous livetime fraction was much smaller during the most intense parts of the burst.

The BATSE data indicate that a first intense spike is followed by a weaker one during the main $1 \mathrm{~s}$ of the burst (Kouveliotou et al. 1994). The question arises whether the same relationship exists between the two spikes as measured by COMPTEL. Any differences would suggest a spectral change taking place from one spike to the next. The first burst events recorded by COMPTEL occur $\sim 100 \mathrm{~ms}$ before the BATSE trigger (Fig. 1, interval A). The exact onset of the burst is uncertain because of the low number of counts.

Individual COMPTEL detector elements assist in determining the relative intensities of the two main spikes. Different D2 detector rates (with an energy threshold of $\sim 450 \mathrm{keV}$ ) are recorded every $2.048 \mathrm{~s}$. A $2.048 \mathrm{~s}$ time boundary fortuitously occurred near the end of the first spike at 1857:11.810 UT (within interval B). Before this boundary, the excess D2 module count rate is attributed to the first $228 \mathrm{~ms}$ of the first spike (A and part of B), while after this boundary the excess is attributed to the remaining $800 \mathrm{~ms}$ of the main $1 \mathrm{~s}$. These excess rates in the single detectors are represented by the two-bin histogram superposed on the telescope count rate. Overlying material makes the detectors relatively immune to adverse dead-time effects caused by the intense $\gamma$-ray flux. Modeling of the individual detectors with an $E^{-2}$ spectrum of $\gamma$-rays indicates that the relative intensities of the two spikes $>450 \mathrm{keV}$ are the same as those measured at lower energies by BATSE. We therefore attribute the low count rate in the first spike as due to unusually large dead-time effects and not due to a much softer spectrum. Based on the count rate of a single D2 detector module, for the $128 \mathrm{~ms}$ before $1857: 11.810 \mathrm{UT}$ we estimate the $>450 \mathrm{keV}$ flux to have an average value of $\sim 200 \mathrm{~cm}^{-2} \mathrm{~s}^{-1}$. Another spike starts approximately at 1857:12.1 UT (D) with structure shorter than $50 \mathrm{~ms}$ as seen in the inset of Figure 1 . After 1857:11.810 UT (C, D, and part of B) the flux $>500 \mathrm{keV}$ is $\sim 16.5 \mathrm{~cm}^{-2} \mathrm{~s}^{-1}$ averaged over the remainder $(800 \mathrm{~ms})$ of the $1 \mathrm{~s}$ burst. The period between the two spikes (C) is consistent with no signal. During the second spike, the intensity is lower, but still large enough to exhaust the capacity of the telemetry buffer at approximately 1857:12.602 UT. Therefore, for the next $\sim 300 \mathrm{~ms}(\mathrm{E})$, no telescope events are recorded. After 1857:13.0 UT all COMPTEL rates are at background levels, and the livetime fraction has returned to $\sim 98 \%$.

The "burst mode" data (Schönfelder et al. 1993) show that in the $2 \mathrm{~s}$ following the burst there is residual emission $>600$ $\mathrm{keV}$ at a level consistent with the measurements reported by Kouveliotou et al. (1994) and also consistent with the lack of events in the COMPTEL telescope data.

We employ a maximum-likelihood technique to determine the position of the burst (de Boer et al. 1992) using telescope data. Twenty-eight events from 0.75 to $30 \mathrm{MeV}$ were selected from the most intense part of the burst (A through D) (Fig. 2) and used to produce an image. Although GRB 930131 was intense, the low instrument livetime and the short duration resulted in fewer recorded events than other bursts observed during the mission (Winkler et al. 1992; Hanlon et al. 1993). This manifests itself in a relatively large statistical error box $( \pm 1.5$ in both directions at the $1 \sigma$ confidence level) around $\alpha_{2000}=12^{\mathrm{h}} 18^{\mathrm{m}}, \delta_{2000}=-9^{\circ} 42^{\prime}$.

We have compared the COMPTEL burst location map to other independent locations by overlaying: the BATSE burst position; the EGRET location contours (Sommer et al. 1994) and the Interplanetary Network annulus (Hurley 1993; Cline, Barthelmy, \& Palmer 1993). Good agreement is obtained between the COMPTEL position and all other locations at the $2 \sigma$ confidence level. Based on COMPTEL data alone, the best location along the triangulation annulus is $\alpha_{2000}=12^{\mathrm{h}} 15^{\mathrm{m}}$, $\delta_{2000}=-9^{\circ} 41^{\prime}\left( \pm 1^{\circ}\right.$ along the annulus). The best location from an image using the combined COMPTEL/EGRET data (Fig. 2) is $\alpha_{2000}=12^{\mathrm{h}} 12^{\mathrm{m}}$ and $\delta_{2000}=-10^{\circ} 21^{\prime}\left( \pm 32^{\prime}\right.$ in both directions at the $1 \sigma$ confidence level).

To construct an energy spectrum we selected 26 events originating within $10^{\circ}$ of the burst position from the first $\sim 1 \mathrm{~s}$ after 1857:11.582 UT (A through D). These events were restricted to a minimum energy of $0.75 \mathrm{MeV}$ and a maximum energy of near $30 \mathrm{MeV}$. Three measured events $>10 \mathrm{MeV}$ compared to a predicted background of 0.1 event establishes the presence of high-energy emission from this burst. The data fit a power-law photon spectrum of $(7.5 \pm 3.1) \times(E / 1 \mathrm{MeV})^{(-1.8 \pm 0.4)} \mathrm{cm}^{-2}$ $\mathrm{s}^{-1} \mathrm{MeV}^{-1}$ (Fig. 3). The largest uncertainty in the spectrum arises from the underrepresentation of the intense part of the 

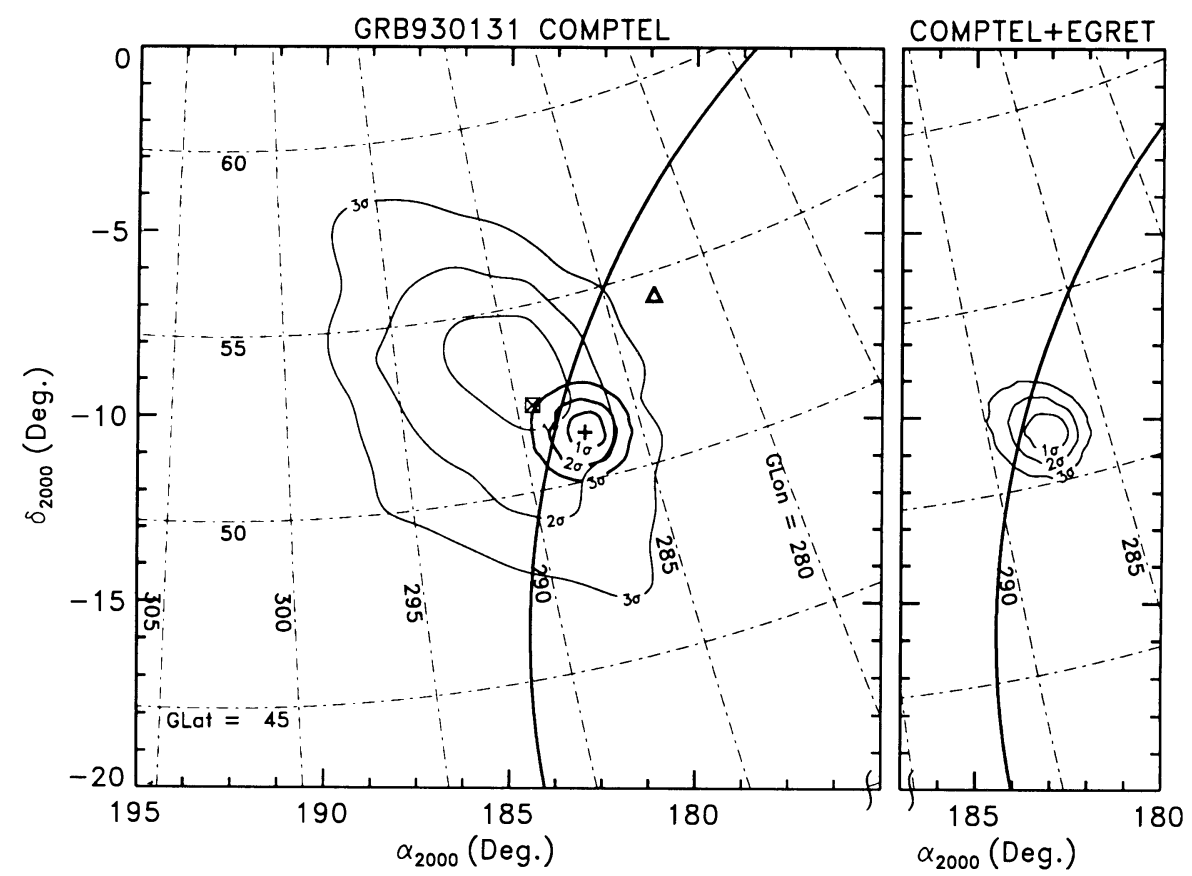

FIG. 2.-(Left): The COMPTEL maximum likelihood ratio map of GRB 930131 in $\alpha_{2000}, \delta_{2000}$ coordinates with a Galactic coordinates grid superposed. Confidence contours of 1,2, and $3 \sigma$ surround the most likely source position (cross). The BATSE location (open triangle), and the EGRET location (plus sign) are shown with the BATSE/Ulysses IPN annulus. Right: An image from the joint COMPTEL/EGRET data (Sommer et al. 1994) with 1,2 and $3 \sigma$ contours.

burst due to livetime effects, affecting only the shape and not the intensity. The spectrum normalization is handled correctly since the instrument integrates the livetime effect over the whole event. The spectra of the two separate pulses within the limits of the statistics show no spectral difference. A preliminary analysis of the high-range "burst mode" data integrated over $1 \mathrm{~s}$, starting at the BATSE trigger, yields a best-fit powerlaw spectrum with an index of $2.2 \pm 0.2$, consistent with the telescope data for the corresponding time interval.

\section{GRB930131 - COMPTEL Telescope Spectrum}

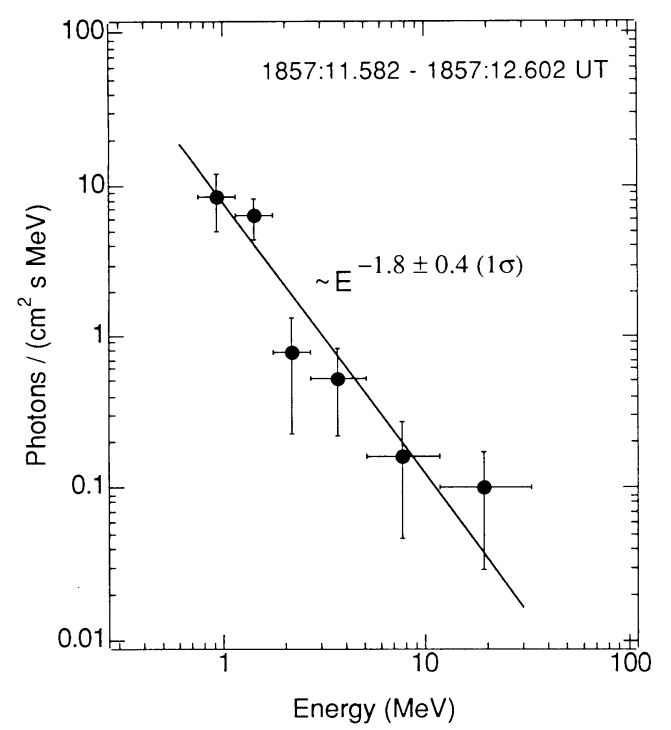

FIG. 3.-The energy spectrum of GRB 930131 . The best-fit power law of $(7.5 \pm 3.1) \times E^{(-1.8 \pm 0.4)} \mathrm{cm}^{-2} \mathrm{~s}^{-1} \mathrm{MeV}^{-1}$ is indicated by the solid line.

\section{DISCUSSION}

The COMPTEL data indicate no significant evolution of the $\gamma$-ray flux during the first $1 \mathrm{~s}$ of the burst. The count rate between the two spikes $(C)$ is consistent with no emission. Although EGRET measures the majority of its high-energy photons between 1 and $30 \mathrm{~s}$ after interval $\mathrm{D}$, this is a result of the EGRET instrumental livetime rather than of spectral hardening (Sommer et al. 1994). The flux measured by EGRET following the intense $1 \mathrm{~s}$ is consistent with the time-integrated COMPTEL spectrum, i.e., $E^{-2}$ (Sommer et al. 1994) but reduced in intensity. This trailing flux folded through the COMPTEL instrument response predicts $\sim 10$ events in the 29 $\mathrm{s}$ after the main burst. Our $2 \sigma$ upper limit on detected "late" events is 12.2 above the background level. Therefore, we find no evidence for spectral hardening in the "delayed" phase of the burst. The EGRET detection at these times presumably results from its greater signal-to-noise ratio compared to that of COMPTEL. Based upon the COMPTEL data above 0.75 $\mathrm{MeV}$, the burst appears to modulate its intensity over a large dynamic range without any significant spectral evolution over the full $30 \mathrm{~s}$ duration of the burst. There is no evidence that the emission $>50 \mathrm{MeV}$ as seen by EGRET is delayed with respect to that at COMPTEL energies.

The intensity $>1 \mathrm{MeV}$ can be used to compute a maximum distance to the burst. Photon-photon interactions attenuate the high-energy (>1 MeV) flux at the source (Schmidt 1978). A maximum diameter $(2 c \Delta t)$ of $30,000 \mathrm{~km}$ can be placed on the burst photosphere (optical thickness $\tau \equiv 1$ at $10 \mathrm{MeV}$ ) based on the $\sim 50$ ms structure measured by the COMPTEL instrument within interval $D$ of the main burst (Fig. 1). At that moment, $\gamma$-rays $>10 \mathrm{MeV}$ were measured by COMPTEL with an instantaneous flux of $\sim 42 E^{-1.8} \mathrm{~cm}^{-2} \mathrm{~s}^{-1} \mathrm{MeV}^{-1}$. This is based upon the measured flux $>450 \mathrm{keV}$ during the last $800 \mathrm{~ms}$ of the intense $1 \mathrm{~s}$, half of which occurred in one $50 \mathrm{~ms}$ 
period (Fig. 1). This value is a lower limit to the instantaneous flux since livetime effects may have limited the count rate at that moment. Therefore, with the intensity and time structure of the burst measured by COMPTEL, we compute a generous upper limit to the burst distance of $250 \mathrm{pc}$ under the assumption of isotropic emission. (A similar figure of $\sim 600 \mathrm{pc}$ can be obtained from the expression of Baring 1994.) In order for a cosmological object $\left(10^{3} \mathrm{Mpc}\right)$ to emit a $\sim 50 \mathrm{~ms}$ spike of such intensity at $1 \mathrm{MeV}$, it must have a Lorentz beaming factor $\gamma_{B}$ greater than the unlikely magnitude of 2800 , radiating into an emission cone smaller than $4 \times 10^{-7} \mathrm{sr}$. If the burst object is within the Galactic halo at a distance of $\sim 30 \mathrm{kpc}$, the lower limit to $\gamma_{B}$ reduces to a value of 10 . Note that the mere existence of high-energy $\gamma$-rays $(>100 \mathrm{MeV})$ does not severely limit the distance. Rapid fluctuations of high-energy emissions are required to limit the source distance. This has been done using the COMPTEL data.

A lower limit to the burst distance can also be computed from the measured parallax of the joint COMPTEL/EGRET image. Any significant deviation from the Interplanetary Network annulus can be interpreted as a parallax effect of the source being nearby. We assume though that the offset of the image centroid from the annulus $(\sim 0.5)$ is indicative of the experimental error. A maximum-likelihood analysis (Connors et al. 1993) using 4.218 AU as the distance from the CGRO to Ulysses and $22^{\circ} .25$ as the annulus radius (Hurley 1993) yields a $2 \sigma$ lower limit of $33 \mathrm{AU}$.

Although these distance limits are crude, they do place realistic constraints on the nature of the burst object. Without significant relativistic beaming, a burst of this intensity is difficult to produce outside the Galaxy, whereas the parallax lower limit on the burst distance does not conflict with heliocentric models (e.g., White 1993). Although a Galactic origin of $\gamma$-ray bursts conflicts with the isotropy and inhomogeneity evidence from the measured burst distributions (Meegan et al. 1992), the measurement of rapid, high-energy bursts such as GRB 930131 also challenge extragalactic and even Galactic halo theories.
The instantaneous $\gamma$-ray luminosity for this object at $250 \mathrm{pc}$, emitting isotropically and integrated from 0.45 to $30 \mathrm{MeV}$ within the $50 \mathrm{~ms}$ spike in interval $\mathrm{D}$ is $3 \times 10^{39} \mathrm{ergs} \mathrm{s}^{-1}$. (Integrating to $1 \mathrm{GeV}$, based on the EGRET measurements, increases this luminosity by only a factor of 2.) Placing the object at $30 \mathrm{kpc}$ and at $10^{3} \mathrm{Mpc}$ with appropriate beaming solid angles implies intrinsic luminosities of $1 \times 10^{41}$ and $1.5 \times 10^{45}$ ergs s $^{-1}$, respectively. Of course, the instantaneous luminosities during interval A could be much larger, perhaps by an order of magnitude. The integrated energy flux for the entire burst from 1 to $30 \mathrm{MeV}$ is $6 \times 10^{-5} \mathrm{ergs} \mathrm{cm}^{-2}$.

\section{CONCLUSIONS}

The intense, short-duration $\gamma$-ray burst GRB 930131 was imaged in the $\mathrm{MeV}$ range by COMPTEL within $7 \mathrm{hr}$ of its occurrence. The COMPTEL localization was found to be consistent with independent BATSE and EGRET locations, as well as the triangulation annulus obtained from BATSE and Ulysses timing data. Multiwavelength observations of the COMPTEL source region have been performed in record time. Spectral analysis indicates that GRB 930131 is a hard event with power-law emission throughout the COMPTEL energy range. The rapid fluctuations of the $>10 \mathrm{MeV}$ flux and its great intensity allow us to constrain the source to be nearby within the Galaxy if we assume that the radiation is not beamed due to relativistic motion. A lower distance limit using an upper limit on the parallax does not conflict with any current models for $\gamma$-ray bursts.

We would like to acknowledge the cooperation of the $C G R O$ Project Staff, the Flight Operations Team, and the BATSE and COMPTEL operations teams for assisting in this Rapid Response Campaign. We also thank Brenda Dingus for her assistance and fruitful discussions. This work was supported in part by NASA contract NAS $5-26645$ and the Deutsche Agentur für Raumfahrtangelegenheiten (DARA) under the grant 50 QV 90968.

\section{REFERENCES}

Baring, M. 1994, ApJS, 90, 899

de Boer, H., et al. 1992, in Workshop on Data Analysis in Astronomy IV, ed. V. Di Gesù et al. (New York: Plenum), 241

Cline, T. L., Barthelmy, S., \& Palmer, D. 1993, IAU Circ., No. 5703

Connors, A., et al. 1993, A\&AS, 97, 75

Greiner, J., et al. 1993, in Compton Gamma-Ray Observatory, ed. M. Fried lander, N. Gehrels, \& D. J. Macomb (AIP Conf. Proc. 280) (New York AIP), 828

Fishman, G. J., et al. 1989, in Proc. of the Gamma Ray Observatory Workshop, ed. W. N. Johnson (Greenbelt, MD: NASA), 2-39

Hanlon, L., et al. 1993, A\&A, in press

Hurley, K. 1993, private communication

Kippen, R. M., et al. 1993, in Compton Gamma-Ray Observatory, ed. M. Friedlander, N. Gehrels, \& D. J. Macomb (AIP Conf. Proc. 280) (New York: AIP), 823
Kouveliotou, K., et al. 1994, ApJ, 422, L59

Meegan, C., et al. 1992, Nature, 355, 143

Ryan, J., Kippen, R. M., \& Varendorff, M. 1993, IAU Circ., No. 5702

Schaefer, B. E., et al. 1994, ApJ, 422, L71

Schönfelder, V., et al. 1993, ApJS, 86, 657

Schmidt, W. K. H. 1978, Nature, 271, 525

Sommer, M., et al. 1994, ApJ, 422, L63

Vanderspek, R., Doty, J. P., \& Ricker, G. R. 1992, in Gamma-Ray Bursts, ed.

W. Paciesas \& G. J. Fishman (AIP Conf. Proc. 265) (New York: AIP), 404

White, R. S. 1993, Proc. Internat. Cosmic Ray Conf., Calgary, 1, 49

Winkler, C., et al. 1992, A\&A, 255, L9 